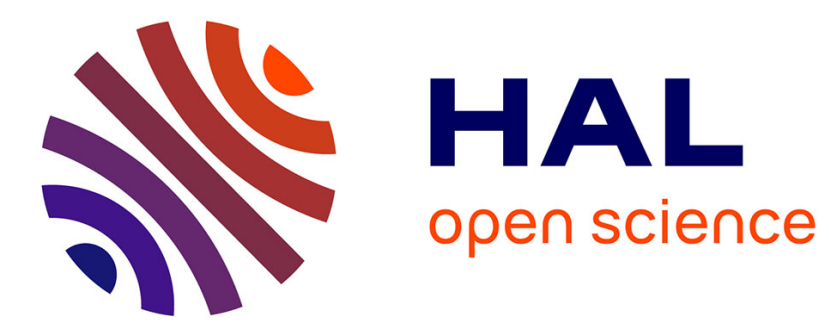

\title{
Le crédit-bail et le principe de substance en France
}

Hélène de Brebisson

\section{To cite this version:}

Hélène de Brebisson. Le crédit-bail et le principe de substance en France. Accountability, Responsabilités et Comptabilités, May 2017, Poitier, France. pp.cd-rom. hal-01907580

\section{HAL Id: hal-01907580 \\ https://hal.science/hal-01907580}

Submitted on 29 Oct 2018

HAL is a multi-disciplinary open access archive for the deposit and dissemination of scientific research documents, whether they are published or not. The documents may come from teaching and research institutions in France or abroad, or from public or private research centers.
L'archive ouverte pluridisciplinaire HAL, est destinée au dépôt et à la diffusion de documents scientifiques de niveau recherche, publiés ou non, émanant des établissements d'enseignement et de recherche français ou étrangers, des laboratoires publics ou privés. 


\title{
Le crédit-bail et le principe de substance en France
}

\author{
Hélène DE BREBISSON
}

Résumé : Dans un contexte d'harmonisation européenne des règles comptables relancé par l'adoption de la Directive 2013/34, le traitement comptable réservé en France au crédit-bail peine à se justifier. L'article fait le point sur le traitement comptable, financier, fiscal et légal de ces contrats pour mettre en évidence l'anomalie que représente l'article 212-5 du PCG, dernier texte à comprendre le crédit-bail comme un contrat de location. Cette étude vise à contribuer à l'amélioration et à la mise en cohérence du cadre réglementaire français sur ce sujet.

Mots-clés : crédit-bail, substance, droit comptable, patrimoine

\begin{abstract}
In the context of European harmonization of accounting rules which was restarted with the adoption of Directive 2013/34, the French accounting treatment of finance leases is hardly justified. This paper reviews accounting, financial, fiscal and legal rules over leases to underline the anomaly represented by article 212-5 in French Chart of Account (PCG), which is the latest text to understand leases as a rental contract. This study aims at contributing to improving French normative environment coherence on this specific issue.
\end{abstract}

Key words: leases, substance over form, accounting rules, patrimony 


\section{Introduction}

La $34^{\mathrm{e}}$ Directive européenne vise à relancer l'harmonisation des référentiels comptables des EtatsMembres, en partant d'un socle commun de principes comptables, parmi lesquels celui de la prééminence de la substance sur la forme (substance over form). Cette directive donne une nouvelle actualité au traitement comptable réservé en France au crédit-bail, exemple type utilisé pour illustrer le principe de substance.

Suivant ce principe la comptabilisation d'une transaction doit en restituer la substance, la réalité économique, et ne pas se limiter à sa forme juridique (voir notamment le cadre conceptuel des IFRS). Dans un environnement anglo-saxon cette démarche confie l'interprétation des transactions au préparateur des comptes, ce qui peut paraître contradictoire avec une vision codifiée de la comptabilité telle qu'on la trouve en France. Peut-être plus difficile à introduire dans un environnement de droit civil, le principe de substance est le seul dans la Directive dont les Etats Membres peuvent exempter les entreprises (Article 6 par. 3). Cette précaution rappelle les difficultés envisagées à propos de l'adoption des normes IFRS dans des pays de droit civil, alors que ces normes sont très influencées par le droit coutumier (common law) (Nobes 2006; Raffournier 2007). Pour autant, les IFRS sont basées sur des principes et non pas des règles, ce qui a été présenté comme un gage de souplesse, facilitant l'intégration de ces normes dans des cadres juridiques divers (Carmona et Trombetta 2008). Par extension, l'adoption de la $34^{\mathrm{e}}$ Directive peut être vue comme celle de principes communs suffisamment généraux pour être adaptables à plusieurs cadres juridiques. Cette tentative d'harmonisation intra-européenne n'aboutira pourtant pas nécessairement à des règles communes, les principes n'étant pas compris de la même manière partout, pour des raisons notamment culturelles et linguistiques (Alexander et al. 2016).

Cette directive renouvelle en France la question de certains traitements comptables. Dans un contrat de crédit-bail, le preneur bénéficie de l'usage d'un bien qu'il loue pendant une période déterminée, à l'issue de laquelle il peut soit, retourner le bien au bailleur soit l'acheter pour une valeur établie à l'avance. Le contrat revêt la forme d'un bail, bien qu'il ait en général en substance les caractéristiques d'un crédit. Cette ambivalence se retrouve d'ailleurs dans le nom donné à cette catégorie bien spécifique de contrat. Si forme et substance diffèrent, alors se pose la question de la meilleure représentation comptable de la transaction : en application du principe de substance, un crédit-bail devrait être enregistré comme un achat financé par un crédit, ce qui permet d'informer le lecteur sur l'actif utilisé par l'entreprise pour ses activités, et sur son niveau réel d'endettement. Mais cette analyse ajoute au bilan un actif dont le preneur n'a pas encore la pleine propriété. Une analyse centrée sur les droits de propriété conduira à privilégier la forme locative du contrat, de manière à présenter le patrimoine juridique de l'entité. Fiscalement les coûts (bénéfices) engendrés par la transaction doivent être déduits (imposés) par (à) l'entité qui les supporte réellement. La question du traitement comptable du crédit-bail renvoie donc à la diversité des objectifs de la comptabilité : quelle priorité donner, de l'objectif légal, fiscal ou informationnel? 
Or aujourd'hui le crédit-bail est analysé selon sa forme en comptes individuels, et selon sa substance en comptes consolidés, suggérant une approche juridique dans les premiers états financiers, économique ou tournée vers l'information dans les seconds. Cette normalisation comptable reflète-t-elle la réalité ? Pour répondre à cette question, nous analysons, dans une approche contextuelle diachronique, les différents aspects de la réglementation, légaux, fiscaux et financiers concernant le crédit-bail pour voir si le crédit-bail y est analysé comme un crédit ou comme un bail : soit ces domaines réglementaires ont des représentations diverses du contrat, que l'analyse comptable différenciée tente de refléter, soit une cohérence d'ensemble émerge, que l'on devrait retrouver dans un traitement comptable unique. Le sujet revêt une nouvelle actualité avec la $34^{\mathrm{e}}$ Directive, qui repose la question de l'applicabilité du principe de substance en France. Les résultats mettent en évidence une anomalie comptable puisque le traitement en comptes individuels du crédit-bail selon sa forme locative ne reflète pas la représentation juridique, financière ou fiscale qui nous semble émerger, où ce contrat est plutôt analysé comme un achat financé. Nous proposons des pistes d'amélioration du référentiel français qui paraissent simples à mettre en œuvre et en mesure de mettre mieux en cohérence les règles comptables avec d'une part la directive européenne, d'autre part l'environnement législatif dans lequel le droit comptable s'insère.

L'article présente les principes comptables applicables dans la réglementation (Section 1) et les débats qu'ils ont fait naître (Section 2) ; le cas du crédit-bail est ensuite analysé dans le détail du point de vue du bailleur et du preneur (Section 3 et 4) ce qui permet de mettre en évidence les éléments de discussion et de conclure.

\section{Les principes applicables dans la réglementation comptable}

\subsection{A l'international}

En Europe, les normes IFRS s'appliquent aux comptes consolidés des sociétés cotées de façon obligatoire $^{1}$. Chaque Etat-Membre est libre d'élargir l'application des normes internationales à d'autres sociétés et/ou aux comptes individuels.

Dans le cadre conceptuel des IFRS le principe de substance over form était décrit comme une des qualités de l'information comptable, permettant d'atteindre l'objectif central d'image fidèle (True and Fair View). Dans le projet de nouveau cadre conceptuel, l'image fidèle est désormais décrite à l'aide de ce principe de substance : "Une image fidèle communique la substance d'un phénomène économique plutôt que de s'en tenir à la forme juridique. Fournir de l'information uniquement sur la forme juridique lorsqu'elle diffère de la substance économique du phénomène sous-jacent ne peut aboutir à une image fidèle. » (IASB 2015). Les débats sur le projet de nouveau cadre conceptuel ont évoqué la redondance entre ces deux principes, l'enregistrement d'une transaction selon sa seule forme ne pouvant jamais conduire à obtenir une image fidèle ${ }^{2}(\mathrm{BC} 3.26$ IASB, 2010).

\footnotetext{
${ }^{1}$ Règlement 1606/2002

2 «Substance over form is not considered a separate component of faithful representation because it would be redundant. » 
[voir situation au Royaume-Uni : le seul principe explicitement donné était longtemps l'image fidèle, le principe de substance paraissait inutile à formaliser car découlant naturellement d'une recherche de l'image fidèle.]

\subsection{En France}

En France les groupes non cotés peuvent opter pour les IFRS ou suivre le règlement CRC 99-02 pour la production des comptes consolidés. Les comptes individuels sont régis par le Plan Comptable Général (PCG). Les textes français ont été influencés par la normalisation internationale depuis l'adoption des $4^{\mathrm{e}}$ et $7^{\mathrm{e}}$ Directives européennes et l'introduction du principe d'image fidèle. Autrefois considérée comme «l'algèbre du droit » (Garnier 1947), la comptabilité française impose aux préparateurs d'être sincères dans l'application des règles pour rapporter les faits et événements de l'entreprise ${ }^{3}$. L'image fidèle a d'abord été ajoutée à cette liste d'objectifs, puis posée comme objectif principal de la production comptable. Aujourd'hui l'image fidèle est le premier principe comptable présenté dans le Plan comptable Général (PCG) (ANC 2014, art 1211). En troisième position sont présentées la régularité et la sincérité, qui imposent de suivre la réglementation en vigueur ainsi qu'une attitude sincère des préparateurs. La régularité est cependant assouplie par la possibilité de déroger à la règle si cela est nécessaire pour obtenir une image fidèle.

Le principe de substance n'est pas formalisé dans le PCG. Pourtant le référentiel intègre de plus en plus une analyse substantielle des transactions. En particulier, le règlement CRC 2004-06 a modifié la définition des actifs qui sont désormais des éléments contrôlés (au lieu de possédés) par l'entreprise ${ }^{4}$. Il s'agissait d'un virageimportant dans la normalisation française, interprété comme substituant la notion de patrimoine économique à celle de patrimoine juridique, et modifiant profondément l'orientation de l'information fournie par le bilan comptable ${ }^{5}$. Pourtant, même si certaines règles suivent le principe de substance, comme le traitement des biens acquis avec une réserve de propriété (Barbe 2012), d'autres règles se limitent à une lecture formelle de la transaction, comme pour le crédit-bail, qui est enregistré en charges pendant la période locative (Art 212-5). La situation ne semble pas nécessairement problématique pour les praticiens (Amblard 2004).

Les comptes consolidés sont régis par un règlement à part, le CRC 99-02. Le règlement ne dresse pas une liste de principes, mais renvoie plutôt sur les principes comptables généraux du PCG (Section II - art 201). L'image fidèle est rappelée régulièrement dans diverses provisions. Le principe de prééminence de la substance est en revanche explicite (Section II para 300), donné comme une des caractéristiques propres à la consolidation. Le terme de substance est plusieurs

\footnotetext{
${ }^{3}$ PCG art 121-3 : "La comptabilité est conforme aux règles et procédures en vigueur qui sont appliquées avec sincérité afin de traduire la connaissance que les responsables de l'établissement des comptes ont de la réalité et de l'importance relative des événements enregistrés. (...)“.

${ }^{4}$ Art. 211-1 : "Un actif est un élément identifiable du patrimoine ayant une valeur économique positive pour l'entité, c'est-à-dire un élément générant une ressource que l'entité contrôle du fait d'évènements passés et dont elle attend des avantages économiques futurs."

${ }^{5}$ www.focuspcg.com/menu_gauche/le_pcg_ou_le_droit_comptable_commun/grandes_reformes/actifs/definition_et_principes 
fois utilisé, dans le cadre d'une analyse substantielle des relations entre les entreprises d'un groupe, pour déterminer l'entrée dans le périmètre et la méthode de consolidation.

\subsection{La $34^{\mathrm{e}}$ Directive}

En 2013 la Directive n³4 impose des principes comptables communs en Europe applicables aux entités hors champ des normes IFRS. Cette Directive a été intégrée dans le PCG par l'Autorité des Normes Comptables (ANC) ${ }^{6}$. Le gouvernement a également mis à jour les obligations comptables du Code de Commerce ${ }^{7}$. Ces textes sont applicables depuis le $1^{\mathrm{er}}$ janvier 2016. Peu de changements sont apportés puisque la plupart des principes énoncés dans l'article 6 paragraphe 1 étaient déjà présents dans le PCG et le Code de Commerce, exception faite du principe de substance.

Concernant ce dernier la Directive prévoit (Art 6 para $1 \mathrm{~h}$ ) : « les postes du compte de résultat et du bilan sont comptabilisés et présentés en se référant à la substance de la transaction ou du contrat concerné ». Notons qu'il n'est pas fait mention de la « forme », la Directive préconisant de se référer directement à la substance de la transaction. Cette phrase est d'ailleurs plus contraignante que sa version anglaise qui utilise l'expression « having regard to the substance » (Alexander et al. 2016). La substance de la transaction est donc la référence de l'enregistrement comptable, mais cette substance n'est pas définie plus avant (Gélard 2013). La Directive met cependant un bémol à l'obligation en autorisant les Etats Membres à exempter les entreprises de cette exigence (Article 6 par. 3). Une remarque préliminaire ( $\left.n^{\circ} 35\right)$ considère enfin que si les principes devraient être les mêmes en comptes individuels et consolidés, les Etats Membres devraient pouvoir les appliquer différemment selon les jeux de compte.

Suite à l'intégration de la Directive dans les règles françaises, le principe de substance est toujours explicite dans le règlement 99-02 et toujours absent du PCG. La situation est donc la suivante : l'article 6 par. 1 (h) n'est pas formellement adopté, ni non plus l'article 6 par. 3 : le principe de substance n'existe qu'en consolidation; dans les comptes individuels, seul un certain nombre de règles respecte une analyse substantielle des transactions. Cela suggère ou bien une intégration du principe au cas par cas, ou que le principe est généralement suivi, sauf exclusion spécifique (Lebrun 2009). Même si la France pouvait se prévaloir de principes différents pour les comptes individuels et consolidés, il n'est pas certain que le PCG soit conforme à la Directive européenne : formellement le principe de substance n'est ni adopté ni rejeté, et sur le fond il n'est pas toujours appliqué, alors que la Directive autorisait d'exempter des entités, pas des transactions.

D'autres pays ont semble-t-il intégré plus facilement le principe de prééminence de la substance (Alexander et al., 2016). Nous relèverons notamment le cas de la Roumanie, où la rupture entre forme et substance gène car elle ne devrait pas exister. C'est en principe le juridique qui a la responsabilité de retranscrire fidèlement la substance des transactions : le principe comptable a donc été introduit, pour être mis en application le moins souvent possible. Soulignons également le cas de l'Allemagne, où le principe est assimilé à un concept utilisé depuis longtemps, l'economic

\footnotetext{
${ }^{6}$ Règlement ANC 2014-03 du 5 juin 2014

${ }^{7}$ Ordonnance 2015-900 
view $^{8}$. Ce principe, aujourd'hui vu comme une méthode d'interprétation téléologique de la loi, confère à la réglementation fiscale le monopole de l'analyse substantielle des transactions. Tout contrat de nature à générer des conséquences fiscales, se voit analyser et définir en substance par l'administration fiscale. Le traitement comptable est déterminé en conséquence, rendant impossible toute dérogation aux règles.

\section{Les débats sur la prééminence de la substance}

L'image fidèle est présentée désormais comme l'objectif des travaux comptables - en comptes individuels et consolidés. Le principe de prééminence de la substance est considéré par la comptabilité anglo-saxonne comme la conséquence directe de cet objectif. Selon le sort réservé à ce principe, le traitement comptable du crédit-bail sera différent : soit on privilégie la forme et le crédit-bail est enregistré d'abord selon sa forme locative initiale ; soit on privilégie la substance, et le bien est activé dès le début du contrat, avec en contrepartie une dette financière.

\subsection{L'image fidèle}

En France, les règles comptables ont été développées après 1945 sous la supervision de l'Etat, en incluant différentes parties intéressées au sujet : représentants des entreprises, professionnels comptables, salariés. Les règles adoptées sont vues comme un compromis permettant de répondre aux besoins des différents utilisateurs de l'information financière, et notamment l'Etat pour la statistique et les impôts, les créanciers et les salariés. Il n'est pas fait mention alors des besoins des actionnaires, du fait d'un marché financier peu développé, peut-être aussi d'une vision des actionnaires impliqués dans l'entreprise et sans doute aussi bien informés que les dirigeants avec lesquels ils se confondent éventuellement. L'autorité des normes comptables s'est accrue lorsque les comptes sont devenus la base de calcul du résultat imposable (1965), puis à nouveau lorsqu'il a été imposé d'enregistrer en comptabilité des écritures purement fiscales, sans réalité économique (Gélard 2012, Tort 2012). Le Plan Comptable a finalement été rendu obligatoire et intégré dans la loi en 1982 (Colasse et Standish 1998). Cet ancrage dans la loi est d'ailleurs relevé par les auteurs qui soulignent qu'auparavant, étant le fruit d'une concertation collective entre toutes les parties prenantes, l'autorité du plan comptable était naturelle. «Cette émergence d'un droit comptable, symbolisé par la loi «comptable » de 1983, allait non seulement rendre plus coercitive la normalisation mais aussi la rigidifier et la rendre moins capable de s'adapter, notamment à l'innovation financière et à la normalisation internationale, et ce au moment où cela allait devenir nécessaire. »

C'est à cette même période que le principe d'image fidèle est introduit dans un tout nouveau « droit comptable » censé s'intégrer dans l'ensemble du système juridique français. La possibilité de déroger à la règle a ainsi pu être considérée comme contraire à la représentation civiliste de la loi (Hoarau 2003). Par ailleurs, l'idée qu'une image fidèle unique puisse répondre aux besoins

\footnotetext{
${ }^{8}$ wirtschaftliche Betrachtungsweise 
d'utilisateurs très divers de l'information comptable a également été discutée. Chaque partie prenante pourrait avoir une vision différente de l'image fidèle de l'entreprise. La normalisation concertée permettait d'établir la réglementation la plus communément acceptable par tous. En instaurant l'objectif d'image fidèle, le débat pourrait ainsi rebondir en étant laissé au niveau des préparateurs des comptes. Delesalle (2014) résume prudemment en considérant que de ces débats est restée l'idée qu'il était possible de présenter une image fidèle parmi plusieurs potentielles, l'objectif étant réputé atteint «dès lors que les règles de la normalisation comptable sont appliquées et respectées ».

En ajoutant un objectif d'image fidèle, le normalisateur français avait trois possibilités selon Colasse (1997) : accentuer le principe de régularité et considérer que le respect des règles devait nécessairement aboutir à une représentation fidèle ; accentuer plutôt sur celui de fidélité, risquant d'affaiblir le principe de régularité. Mais, sans réelle définition de l'image fidèle ni remise en cause des règles existantes, le normalisateur a finalement eu une stratégie " d'évitement » : la fidélité devait guider la pratique comptable lorsqu'une règle était manquante ou insuffisante pour représenter la réalité d'une situation. Le principe de prééminence de la substance sur la forme n'a pas été introduit, qui aurait consacré la présentation d'un patrimoine économique plutôt que purement juridique. Le résultat fut un accroissement de l'usage des notes annexes.

Le cadre conceptuel des IFRS, décrit l'image fidèle comme un objectif, car elle permet de fournir une information utile à la prise de décision. L'image est fidèle lorsque les descriptions sont données de façon complète, neutre et exempte d'erreurs (IASB 2010). Or le problème est posé différemment en France : le débat porte sur l'ambivalence entre une présentation dite économique du patrimoine de l'entreprise, et une présentation juridique, c'est-à-dire basée sur les droits de propriété.

\subsection{Le principe de substance}

L'accession de la normalisation comptable au rang de droit comptable impose une cohérence des textes avec l'architecture juridique d'ensemble. Or la notion de substance est en droit très centrale. On citera notamment l'article 1156 du Code Civil (1804) : «On doit dans les conventions rechercher quelle a été la commune intention des parties contractantes, plutôt que de s'arrêter au sens littéral des termes ». Un contrat devrait exprimer la substance de la volonté des parties, la forme devrait e pratique exprimer la substance. Ainsi le principe comptable est-il vu comme redondant avec le principe civil. Reste à savoir s'il faut inscrire formellement ce principe dans les règles comptables afin de guider la traduction comptable des analyses juridiques, ou si la redondance des principes peut conduire à des problèmes d'interprétation.

Par ailleurs le premier rôle de la comptabilité n'est pas nécessairement le même selon les disciplines. En comptabilité, une des premiers rôles mis en avant est de produire une information utile à la prise de décision, en particulier de gestion. Cette intuition est nourrie par l'influence de la normalisation internationale, mais aussi par le processus de normalisation en France qui intègre une représentation de l'ensemble des parties prenantes. Sur le plan légal la comptabilité a également diverses utilités dont la distribution de dividendes, ou en cas de litige la preuve de 
réalisation de transaction, ou le calcul du patrimoine en vue d'une liquidation. Selon que l'on cherche une information économique ou une preuve légale, l'établissement du patrimoine de l'entreprise ne sera pas le même.

Les juristes français ont réagi à cette opposition entre deux visions de la comptabilité, l'une dite anglo-saxonne autour de l'évaluation d'un patrimoine économique et l'autre à la française centrée sur un patrimoine juridique. Ils contestent l'idée « que le droit ne décrit pas l'ensemble des situations dont il est censé rendre compte ou, du moins, qu'il le fait d'une manière trompeuse. » (Pasqualini 1992). Il est donc dangereux de considérer que le droit ne rendrait pas compte de l'économique, et les deux termes ne devraient pas être ainsi opposés. Le patrimoine est quant à lui défini comme « une universalité juridique, laquelle est constituée par un ensemble de droits et d'obligations, c'est-à-dire d'actif (les droits) et de passif (les obligations), se rapportant à l'activité d'une personne, l'actif répondant du passif » (Larroumet 1984). Cette position est également développée par Medus (1999), qui considère que le bilan doit présenter un patrimoine fondé sur les droits de propriété, et non pas une situation patrimoniale définie comme une notion financière qui ne serait pas intégrée dans notre droit. L'auteur considère que l'image fidèle peut être représentée à l'aide du bilan - le patrimoine - complété par l'annexe - les engagements complémentaires - le tout représentant la situation patrimoniale de l'entreprise.

Sériaux (1994) discute le concept en excluant du patrimoine juridique les biens sous possession précaire ou provisoire. Il souligne par ailleurs que « les dettes ne sont rattachables qu'à la personne de l'obligé et non à ses biens ». Le patrimoine dans sa définition courante ne fait état que des biens possédés par le titulaire, non de ses dettes : même si le titulaire utilisera ses biens pour répondre de ses dettes, il est libre d'affecter ses ressources comme il l'entend.

Raybaud-Turrillo (1995) rejette également l'opposition faite entre substance et forme d'un contrat, mais considère que l'analyse substantielle des transactions est en fait une analyse juridique. « La finalité de l'analyse substantielle est (...) de mettre en évidence les contradictions ou les ruptures de cohérence qui apparaissent au sein des catégories juridiques formelles, en raison des mutations qualitatives apparues dans les faits. (...) elle peut ainsi conduire à proposer de nouvelles catégories juridiques. » Sa proposition rejoint les propos de Farjat (1986). Lagarrigue (1983) redéfinit de la même manière la substance économique : « Le principe dit du primat de la réalité sur l'apparence n'est pas (...) la prétendue prééminence de la réalité économique sur l'apparence juridique, mais (...) la nécessité de rechercher la substance et la portée effective des dispositions juridiques derrière et au-delà de l'habillage formel qui est le leur. » Il n'y aurait donc pas opposition entre deux réalités, mais une seule réalité des engagements pris par les parties et leurs conséquences dans les faits. La comptabilité doit bien présenter un patrimoine juridique, ce dernier devant refléter la substance des dispositions juridiques dans lesquelles l'entité est effectivement engagée.

Ce débat juridique ravive différentes questions comptables fondamentales, en particulier celle du critère de comptabilisation et celle de l'engagement. Sur le critère de comptabilisation, certains auteurs s'arrêtent à la notion des droits de propriété acquis quand d'autres tiennent aussi compte de droits partiels - d'usage ou d'usufruit, en vue d'une acquisition complète in fine. Ce point nous renvoie à la comptabilité d'engagement. Peut-on enregistrer un actif dès lors que l'on a pris des engagements tels qu'il est fort probable qu'en fin de contrat on en soit complètement propriétaire ? 
Selon le principe d'engagement les transactions sont enregistrées dès lors qu'elles sont acquises (recettes) ou engagées (dettes) et même si la contrepartie financière (paiement) est prévue à une date ultérieure. L'analyse de la portée effective des dispositions juridiques implique de déterminer le moment de l'engagement irrévocable des parties. Cela revient à présenter dès le début d'un contrat, et sous l'hypothèse qu'il se déroule comme prévu - soit pourrait-on dire sous l'hypothèse de continuité d'activité, l'ensemble de ses conséquences.

Le principe de prééminence de la substance reviendrait à traduire en termes comptables les engagements juridiques pris dans leur ensemble, qu'ils soient explicites ou non, et leurs conséquences effectives, par opposition à une vision partielle de ces obligations telle que prises à une étape du contrat observée indépendamment des autres. Concernant un actif qui sera en fin de contrat probablement propriété de l'entreprise, le fait de l'enregistrer dès qu'il est sous contrôle est traduit par l'idée d'une présentation d'un patrimoine économique. Ceci peut être interprété comme d'un patrimoine juridique dans lequel certains titres de propriété sont encore conditionnels, mais suffisamment probables pour être enregistrés. Le terme " économique » illustre l'idée que l'actif est déjà utile à l'entreprise, avant même la finalisation de l'échange formel de propriété. Dans un crédit-bail, la transaction complète revient à un engagement comparable à un prêt l'argent prêté étant remplacé par un bien - alors que, pris étape par étape, ce contrat s'assimile à un bail. Bien que le preneur ne puisse être considéré comme propriétaire du bien, il en a l'usage, et fait sien les fruits de l'utilisation du bien.

\subsection{Conséquences sur le crédit-bail}

Curieusement, si l'image fidèle a été débattue par les auteurs comptables, il est peu fait mention d'une doctrine ou d'une autre concernant le principe de substance. Tout juste peut-on comprendre ici ou là un certain regret de n'avoir pas mené la logique de convergence jusqu'au bout. En revanche le débat sur la substance a davantage mobilisé les juristes.

Pasqualini (1992) ou Medus (1999) se concentrent sur une certaine défense du droit et de sa mission de formalisation du réel. Mais, s'appuyant sur une vision juridique de la comptabilité chargée d'évaluer le patrimoine basé sur les droits de propriété, ils restent attachés à un traitement du crédit-bail selon sa forme locative tant que le bien n'est pas définitivement acquis. Sériaux (1994), en montrant que le passif n'est pas affecté aux actifs, et que les dettes sont éteintes selon la libre action du titulaire, se positionne également indirectement en faveur d'une lecture formelle du crédit-bail. En effet dans un crédit-bail, la dette est directement rattachée au bien : en cas de procédure collective, la présentation d'un bilan comprenant un crédit-bail pourrait prêter à confusion. Le bien loué étant directement lié au passif correspondant, les deux items devraient être exclus des calculs de valeurs liquidatives d'actif et de passif exigible.

Le raisonnement tenu par Raybaud-Turillo (1995) amène à enregistrer le crédit-bail selon sa substance, sa dimension financière. Si l'on poursuit, le crédit-bail pourrait même être considéré comme une catégorie juridique à part, le nom étant d'ailleurs créé pour une catégorie de contrat bien identifiée. Il s'agit donc non pas de prioriser en comptabilité la forme ou la substance de ce contrat, mais d'associer à cette catégorie juridique identifiée, le juste traitement comptable. 
Dans un cas le patrimoine présenté au bilan s'arrête aux droits de propriété acquis, reléguant en annexe toute autre information complémentaire. La position souffre cependant d'un manque de pragmatisme et pose la question de l'utilité d'un tel bilan. Est-ce une information utile pour le lecteur cherchant à comprendre la performance de l'entreprise (Alexander 1997) ? L'annexe estelle lue avec autant d'attention que le bilan ? Colasse (1997) pointe le danger d'une annexe pléthorique qui risque de ne pas améliorer la compréhension du lecteur, et même de devenir l'outil d'une véritable stratégie de désinformation.

Dans l'autre, on considère que le crédit-bail est en réalité une catégorie juridique à part qu'il convient de traiter comptablement pour ce qu'elle représente, c'est-à-dire un prêt affecté. Cette position a semble-t-il séduit les auteurs en comptabilité, qui citent régulièrement l'article de Raybaud-Turillo (1995) dans leurs travaux. Un tel raisonnement renvoie à la fois aux positions prises par la Roumanie et l'Allemagne : elle renforce le droit en soulignant qu'il doit exprimer la substance d'une transaction et qu'il ne devrait en principe pas exister de différence entre substance et forme. Elle renforce également l'importance de la règle et l'hypothèse selon laquelle le respect des règles devrait permettre $d$ 'obtenir une image fidèle.

La comptabilité jouerait alors son rôle d'information en améliorant la comparabilité entre entités dont les investissements sont réalisés selon des formes différentes : le patrimoine économique rend compte de l'ensemble des ressources nécessaires aux activités de l'entreprise, quand le patrimoine juridique s'arrête à ce qu'elle détient, reléguant en annexe les autres engagements pris ou donnés. Dans ces débats, il semble que les comptables soient tentés par l'analyse en substance du créditbail, bien que mal à l'aise sur la capacité ou non du principe de substance à s'intégrer dans l'arsenal juridique français. Les juristes semblent voir la production comptable dans un rôle strictement juridique, réfutant une vision économique de chiffres donnés pour une portée uniquement légale.

La recherche en finance s'est aussi intéressée au crédit-bail, à la suite de Ang et Peterson (1984) qui, constatant qu'un niveau croissant de crédit-bail s'accompagnait en fait d'un niveau croissant de dette, mettent en avant un leasing puzzle. Le crédit-bail a été comparé au crédit classique en termes de coûts d'agence et d'aléa moral, et les taux accordés ont fait l'objet d'études pour comprendre le niveau de risque perçu (par exemple Lease, McConnell, et Schallheim 1990; De Bodt, Filareto, et Lobez 2000; Lobez et Statnik 2007). Le consensus semble aujourd'hui admettre que le crédit-bail puisse être un substitut ou un complément à la dette (Schallheim, Wells, et Whitby 2013) : pour un profil moyen c'est un complément, qui devient substitut lorsque le profil est trop risqué. Dans cette littérature, il ne fait aucun doute pour les financiers qu'il s'agit bien d'un contrat de financement, lu donc dans sa substance.

\subsection{Question de recherche}

L'environnement réglementaire français semble s'orienter vers une analyse substantielle des transactions, ce qui n'est pas nécessairement incompatible avec un cadre juridique de droit civil ou continental, dans lequel le droit a dès le départ vocation à formaliser la substance des relations contractuelles. La grande différence entre droit coutumier et civil à cet égard tient dans l'origine 
de l'analyse substantielle : cette analyse est laissée à l'individu dans le droit coutumier, alors qu'elle est centralisée par une autorité réglementaire dans le droit civil.

Pour autant l'établissement en comptabilité d'un patrimoine économique ne fait pas l'unanimité. Orienter la comptabilité dans la présentation d'un patrimoine établi selon les droits de propriétés acquis ou selon un contrôle opérationnel nécessite de choisir parmi les objectifs de la comptabilité - légaux, fiscaux, d'information financière, un axe prioritaire. Dans un objectif légal, il n'est pas question de confondre le patrimoine du preneur et du bailleur. Dans un objectif fiscal, il s'agit de répartir le coût de l'emprunt et de l'usage du bien sur les parties qui le supportent. Dans un objectif financier, il s'agit de présenter un niveau de dette réellement engagé. Enfin dans une perspective plus large, la question est de savoir si le traitement comptable actuel est en cohérence avec le traitement juridique, économique, financier ou fiscal qui est fait de ce contrat.

Nous analysons maintenant plus en détail le traitement du crédit-bail du point de vue du preneur (section 3) et du point de vue du bailleur (section 4) pour comprendre l'orientation générale de la réglementation juridique, fiscale, financière et comptable et analyser la cohérence d'ensemble.

\section{Le traitement du crédit-bail du point de vue du preneur}

\subsection{Economie et droit du contrat}

Les dispositions courantes du crédit-bail impliquent pour le preneur du bien un engagement ferme sur la durée du contrat, sans possibilité de sortie ou avec des conditions de sortie coûteuses. Le preneur n'est pas propriétaire du bien pendant le contrat, mais il en a l'usage et la possibilité de personnalisation. La maintenance est en général à sa charge. A la fin du contrat une option d'achat lui permet de retourner le bien ou de le conserver moyennant un prix connu d'avance, en général assez faible. Par ailleurs, tant qu'il honore ses engagements, le preneur ne peut se voir retirer l'actif des mains : si le propriétaire légal cède le bien en cours de contrat, le contrat se poursuit ${ }^{9}$. En conséquence, dans la mesure où la réalisation du contrat reste probable, le preneur d'un bien en crédit-bail a un droit d'usage du bien tel qu'il en est quasi propriétaire.

Sur le plan économique un preneur de crédit-bail tient compte de ce type d'engagement dans le calcul de son endettement réel. C'est en tout cas ce qu'en pratique réclame le banquier pour calculer le risque crédit de son client (Bacqueroët 2010).

On relèvera par ailleurs le cas du preneur public pour qui la demande de crédit-bail ne relève pas des demandes d'investissement, mais du Code des Marchés Publics de Fournitures. Ce code définit la procédure à suivre concernant l'achat, la location, la prise en crédit-bail ou la location-vente de produits. Il n'est pas fait de différence entre les modalités de prise de possession des biens, l'évaluation se faisant sur l'ensemble de fournitures faisant l'objet du marché (article 27). Cette

\footnotetext{
${ }^{9}$ Art 313-8 du Code Monétaire et Financier : «En cas de cession de biens compris dans une opération de crédit-bail, et pendant la durée de l'opération, le cessionnaire est tenu aux mêmes obligations que le cédant qui en reste garant. » 
démarche semble neutraliser les formes contractuelles possibles de l'acquisition de fournitures, évoquant une analyse substantielle du contrat de crédit-bail.

\subsection{Traitement fiscal}

Sur le plan fiscal, les crédits-baux permettaient d'optimiser l'ancienne Taxe Professionnelle (TP), en réduisant la valeur brute des actifs de l'entreprise. Mais la réforme de 2012 a remplacé la TP par deux taxes, dont la Contribution Foncière des Entreprises (CFE) fondée sur les bâtiments et terrains contrôlés par l'entreprise. L'intérêt fiscal pour le preneur de crédit-bail a sur ce point disparu.

Pour le calcul de l'Impôt sur les Sociétés (IS), le traitement fiscal actuel suggère une analyse substantielle des contrats de crédit-bail (Editions Francis Lefebvre 2016). Par exemple la part de redevance représentative de l'amortissement d'un terrain est non déductible (Barbe 2012). La redevance liée aux véhicules de tourisme est plafonnée pour tenir compte de la valeur du véhicule, par analogie avec le cas de l'amortissement d'un véhicule acquis. Mieux, les redevances de créditbail entrent dans le cadre de la limitation générale de déductibilité des charges financières pour la partie équivalente aux charges d'intérêt (CGI Art 212-bis). Enfin, pour un crédit-bail immobilier, le prix d'acquisition de l'immeuble est recalculé comme s'il avait été acquis dès le départ : on ramène ainsi le bien à la valeur nette théorique en enregistrant la valeur initiale du bien diminuée des amortissements qui auraient dû être pratiqués (Editions Francis Lefebvre 2016). C'est une situation particulière où le fisc impose un retraitement extra-comptable avec revalorisation fiscale d'un bien, alors que l'habitude était plutôt d'imposer des écritures fiscales en comptabilité. Comme le souligne (Rossignol 2007), le principe comptable de prééminence de la substance sur la forme est source d'inspiration pour le juge de l'impôt, qui peut ainsi mieux comprendre les contrats pour améliorer les règles fiscales.

\subsection{Traitement comptable}

En comptes consolidés, l'entité suivra ou bien le règlement 99-02 ou bien les normes IFRS. Les conditions courantes du crédit-bail le rapprochent en général d'un contrat de location financement, retraité de façon obligatoire en IFRS, préférentielle en 99-02. Le preneur devra donc faire apparaître au bilan un actif ainsi qu'une dette financière dès le début du contrat. Si le preneur produit ses comptes consolidés en normes IFRS, à partir de 2019, en vertu de la norme IFRS 16 le schéma évoluera. Quelles que soient les conditions du crédit-bail, son retraitement fera apparaître un actif immatériel lié à un droit d'usage du bien, ainsi qu'une dette. Si le financement est toujours visible, ce n'est plus le bien qui est présenté à l'actif, mais son droit d'usage : cela peut rendre difficile une lecture opérationnelle des actifs de l'entreprise, mais facilite la distinction entre actifs possédés et contrôlés.

En comptes individuels le preneur d'un crédit-bail enregistre une charge d'exploitation pendant la période locative, puis active le bien à l'issue du contrat s'il a levé l'option d'achat, pour la valeur de cette option, suivant les dispositions du PCG (art 212-5). Il y a donc une rupture entre 
l'information fournie par les deux jeux de comptes. Le traitement prévu en comptes individuels a de quoi surprendre, notamment après la réforme de 2004 puis l'adoption de la Directive 34.

Une explication possible au traitement comptable formel du crédit-bail pourrait venir du rôle légal de la comptabilité, utilisée comme preuve de la réalisation d'un contrat : une charge de location pourrait être plus facile à lire et à réconcilier à la facture sous-jacente. La simplicité a longtemps été considérée comme une des qualités du système comptable français (Hoarau 2003, p45). Cela étant, sans renier la difficulté technique de l'activation d'un crédit-bail, l'actif corporel qu'il fait apparaître est au moins aussi visible qu'une charge de location. L'argument ne tient donc pas, sauf à douter des compétences des préparateurs de comptes. La rupture entre les deux jeux de comptes impose une contrainte au préparateur et au lecteur.

\subsection{Conséquences}

Du point de vue du preneur le contrat est traité en substance comme un achat financé dans les comptes consolidés, et sur le plan économique. De même le fisc suit une analyse de plus en plus substantielle, en particulier au travers du plafonnement des intérêts financiers. En revanche les comptes individuels conservent une analyse formelle du contrat.

Ainsi l'information financière donnée par les comptes individuels est-elle trompeuse sur le créditbail : le bilan ne fait pas apparaître le niveau réel d'endettement de l'entreprise. Finalement pour des entités simples qui ne produisent pas de comptes consolidés, le traitement comptable du créditbail peut conduire à des erreurs de gestion de la part de décisionnaires qui ne sont pas nécessairement des financiers. Or l'entité juridique simple représente le cas le plus courant, où les comptes individuels ont un rôle informatif majeur.

\section{Le traitement du crédit-bail du point de vue du bailleur}

\subsection{Le contrat}

Les contrats de crédit-bail sont régis par le Code Monétaire et Financier (art. L 313-7) et réservés aux sociétés de financement. Comme pour la location longue durée ou la location avec option d'achat réservée aux particuliers, le crédit-bail est un exemple de financement affecté, présenté sans doute possible comme un crédit (voir par exemple sa classification en NAF 64.91Z par l'Insee, dans les activités des services financiers). Le crédit-bailleur est donc une institution financière relevant de l'Autorité de Contrôle Prudentiel et de Résolution (ACPR).

Le bailleur est le propriétaire légal du bien, mais n'en a pas la jouissance. En particulier il ne peut le vendre en cours de contrat qu'à un autre crédit-bailleur, afin d'assurer la continuité du contrat. La propriété légale ne sert finalement que de garantie puisque le bien a vocation à être acheté par le preneur au terme du contrat, ou revendu en cas de retour, ou en cas de défaut du preneur. 


\subsection{Traitement prudentiel}

En 2014 les règles de solvabilité en France s'appuient sur les comptes individuels et n'intègrent qu'un risque résiduel sur les contrats de crédit-bail (Ordonnance du 5 mai 2009 modifiée par l'ordonnance du 3 Nov 2014). En effet seuls les concours restant à courir sur moins d'un mois sont pris en compte dans le calcul des actifs risqués. Cette interprétation diffère de ce qu'on peut trouver dans l'étude de Schmit (2004), qui considère qu'en traitant le crédit-bail comme un crédit classique non garanti, l'approche standard de Bâle II impose une couverture excessive du risque.

Suivant les préconisations de Bâle III, les calculs prudentiels devraient être réalisés sur la base des comptes consolidés, et non plus individuels (ACPR 2016). Les actifs mis en crédit-bail seront valorisés à l'actualisation des paiements minimaux, et traités, sous conditions, en prêts garantis (pondérés à $75 \%$ en approche standard). Si l'exposition est une valeur résiduelle de l'actif loué, l'exposition pondérée est évaluée à $100 \%$ de la valeur résiduelle divisée par le nombre d'années restant à courir. On voit donc une évolution de la réglementation prudentielle et son interprétation en France vers une approche substantielle de ces contrats.

\subsection{Traitement fiscal}

Sur le plan fiscal le bailleur peut opter pour un régime d'amortissement financier ou le régime de droit commun. Selon le régime d'amortissement financier l'ensemble des biens loués est amorti «sur la durée des contrats correspondants, au rythme de l'amortissement du capital engagé pour l'acquisition de ces investissements répercuté dans les loyers » (Editions Francis Lefebvre 2016). En pratique, cela revient à suivre le plan d'amortissement du financement sous-jacent au contrat.

En droit commun, l'amortissement fiscal des composants se fait selon les durées d'utilisation réelle, mais les bailleurs n'ont pas à décomposer les biens si la charge de renouvellement des composants ne leur incombe pas. On peut donc supposer que les biens sont en pratique rarement décomposés par l'institution financière. Pour les autres biens, les durées d'amortissement sont l'utilisation réelle ou bien les durées d'usage admises avant 2005 (Editions Francis Lefebvre 2016, par. 9205 et s.). Par exemple les usages courants d'amortissement fiscalement déductibles sont pour le matériel 7 à 10 ans, 4 à 5 ans pour les matériels roulants. Si le bailleur choisit le régime de droit commun, le mode d'amortissement pourra être au choix le linéaire ou le dégressif si le bien loué est admissible. Par ailleurs le bailleur peut aussi déduire une provision équivalente à la différence entre la valeur résiduelle du bien en fin de contrat et l'option d'achat si celle-ci est inférieure. Ainsi, l'éventuelle perte comptable constatée en fin de contrat à la cession du bien est fiscalement anticipée.

Prenons l'exemple d'un matériel de transport, par exemple un navire, dont la durée réelle d'utilisation sera de 15 ans. Un contrat de crédit-bail peut être signé sur cette durée, et le bailleur pourra choisir entre un régime d'amortissement financier, un régime de droit commun linéaire (sur la durée d'utilisation) ou dégressif (sur 8 ans). Si l'on compare les 3 régimes d'amortissement possibles, c'est l'amortissement dégressif qui permet de réduire au maximum la perte financière liée à l'impôt société (voir annexe 1). En outre, pour un matériel d'une valeur initiale de 100000 
euros, le crédit-bailleur crée dans les 4 premières années un déficit fiscal cumulé de 12000 euros. Si l'on compare plusieurs durées possibles d'amortissement pour une même durée de contrat sur 15 ans, il apparaît que l'intérêt pour l'amortissement dégressif augmente à mesure que la durée d'amortissement fiscal se réduit.

\subsection{Traitement comptable}

Sur le plan comptable le crédit-bailleur dans les comptes individuels conserve à son actif le bien jusqu'à la levée d'option par le preneur. Dans les comptes consolidés le contrat sera retraité pour faire apparaître un financement, que ce soit en normes IFRS actuelles ou futures, ou selon la méthode préférentielle du règlement 99-02.

Le traitement comptable est donc symétrique pour le preneur et le bailleur, avec la même rupture entre comptes individuels et consolidés.

\subsection{Conséquences}

Ainsi, le traitement comptable est-il en faveur du crédit-bailleur : il présente en comptes individuels un actif tangible plutôt qu'un prêt, ce qui allège pour l'instant les problématiques de solvabilité ; il peut bénéficier d'un amortissement dégressif ce qui génère en début de contrat un déficit fiscal alors même que le contrat crée de la valeur. Sur le plan fiscal soulignons le fait que l'analyse du contrat n'est pas symétrique entre le preneur et le bailleur : alors que pour un preneur de nombreuses dispositions poussent au retraitement en substance du contrat, du point de vue du bailleur toutes les situations sont possibles, laissant le bailleur traiter ce contrat façon formelle ou en substance : soit l'actif sous-jacent est amorti comme le serait un prêt, soit il est amorti comme le serait l'actif réel.

\section{Conclusion}

Notre étude visait à étudier la réglementation française concernant le contrat de crédit-bail, du point de vue du preneur et du bailleur, pour comprendre les enjeux de cette réglementation pour les deux parties. Les résultats montrent que le crédit-bail est traité en substance, comme un crédit affecté, sur le plan économique et prudentiel, ainsi qu'en comptes consolidés. La qualité du bailleur comme institution financière pousse également à une analyse juridique de ce contrat comme d'un contrat de financement. Le traitement fiscal est quant à lui asymétrique avec une analyse plutôt substantielle du contrat côté preneur, et une analyse s'appuyant sur les droits de propriété côté bailleur. Le bénéfice de l'amortissement dégressif lié au bien est réservé au bailleur, alors même que le preneur subit le plafonnement des charges d'intérêt financier. Dans les comptes individuels, le traitement locatif est aujourd'hui obsolète par rapport aux nouvelles réglementations prudentielles. Il présente également un défaut d'information réel, en particulier auprès des petites entités qui ne produisent que des comptes individuels. 
Le droit comptable évolue vers une vision dite économique du patrimoine, le principe d'image fidèle suggérant que l'on présente l'ensemble des ressources utilisées par l'entreprise pour sa création de valeur. Si cette vision est considérée comme anglo-saxonne, elle ne paraît pas contradictoire avec le droit romain puisque notre Code Civil enjoint déjà à lire dans les contrats au-delà de la forme pour y trouver la substance de l'accord. L'article 212-5 du PCG paraît donc incohérent non seulement avec l'orientation générale des règles comptables, mais aussi avec le Code Civil, et même dans une certaine mesure avec les règles fiscales qui ont évolué sur ce point. En outre le traitement comptable actuel n'informe pas correctement l'utilisateur des comptes individuels sur l'endettement financier réel de l'entreprise. Or pour l'écrasante majorité des entreprises, il n'y a pas de comptes consolidés, et donc pas d'autre source d'information financière normée. La situation n'est finalement qu'au bénéfice des banques, sur le plan fiscal principalement. On comprend mieux dès lors que les institutions financières défendent un schéma d'écriture « formel », comme il a été rappelé lors d'une table ronde sur le principe de substance lors des $5^{\text {è }}$ Etats généraux de la Comptabilité organisés par l'ANC ${ }^{10}$. Bien que les banques françaises soient en pointe sur le marché du crédit-bail, doit-on craindre qu'un changement de norme comptable puisse assécher l'économie ?

L'évolution des normes françaises a suscité un certain nombre de critiques, notamment concernant sa complexité (Tort 2012), ou la rupture de continuité entre les comptes individuels et consolidés (Hoarau 1995) qui conduit à des difficultés pratiques (Simon 2010; Senicourt 2012). Par ailleurs, la comptabilité était autrefois basée sur un compromis entre les parties prenantes autour d'un objectif légal et d'information. Mais l'influence croissante du fisc sur les règles comptables a conduit notamment à enregistrer des éléments purement fiscaux dans les comptes, et tend à détourner la comptabilité de son objectif initial (Lopater 2015).

Cette étude propose des pistes pour améliorer le cadre réglementaire française en défendant une cohérence d'ensemble. Les expériences voisines de Roumanie ou d'Allemagne semblent montrer qu'une intégration du principe de substance en droit civil est possible, comme d'ailleurs certains juristes semblent le montrer. Une réforme du principe de substance et des règles aujourd'hui basées sur l'analyse formelle des transactions permettrait une amélioration de la cohérence d'ensemble des règles comptables, et pourrait se faire dans un cadre civiliste. Ces travaux devraient être complétés par une analyse plus large des engagements aujourd'hui encore classés hors bilan. Les conséquences de l'intégration du principe de substance portent également sur d'autres engagements aujourd'hui hors bilan, qu'il faudrait probablement réintégrer. Il serait également intéressant de comprendre comment ce traitement comptable a pu perdurer, malgré les évolutions réglementaires européennes et une convergence comptable internationale croissante.

\footnotetext{
${ }^{10}$ Table ronde sur le «principe de substance over form », position développée par F. Palle Guillabert (Association Française des Sociétés Financières, ASF), 5è Etats Généraux de la Recherche Comptable, ANC, 11/12/2015, Paris.
} 


\section{Références}

\subsection{Références normatives}

ACPR. 2016. Notice - Modalités de calcul des ratios prudentiels dans le cadre de la CRD IV. Banque de France. https://acpr.banque-france.fr/

ANC (1999). Règlement CRC-99-02. France. http://www.anc.gouv.fr/

ANC (2014). Règlement 2014-03. Plan comptable général. France. http://www.anc.gouv.fr/

Code de commerce. http://www.legifrance.gouv.fr/

IASB. 2010. Conceptual Framework for Financial Reporting 2010. http://www.ifrs.org/.

IASB. 2015. Exposure Draft: Conceptual Framework for Financial Reporting. http://www.ifrs.org/.

Ministère de l'Economie (2009). Arrêté du 5 mai 2009 relatif à l'identification, la mesure, la gestion et le contrôle du risque de liquidité. http://www.legifrance.gouv.fr/

European Parliament and Council (2013). Directive 2013/34/EU of the European Parliament and of the Council of 26 June on the annual financial statements, consolidated financial statements and related reports of certain types of undertakings, amending Directive 2006/43/EC of the European Parliament and of the Council and repealing Council Directives 78/660/EEC and 83/349/EEC. Version française disponible sur : http://eur-lex.europa.eu/legalcontent/FR/TXT/HTML/?uri=CELEX:32013L0034

Editions Francis Lefebvre. 2016. Fiscal, 2016. Levallois-Perret : Éditions Francis Lefebvre.

\subsection{Publications scientifiques}

Alexander, David. 1997. Stability, pseudo-stability, information and pseudo-information. European Accounting Review 6 (4): 755- 66.

Alexander, David, Hélène Brébisson (de), Cristina Circa, Eva Eberhartinger, Roberta Fasiello, Markus Grottke, et Joanna Krasodomska. 2016. Philosophy of Language and Accounting. In 12th Conference on European Financial Reporting, September. Fribourg.

Amblard, Marc. 2004. Conventions et comptabilité : vers une approche sociologique du modèle. Comptabilité - Contrôle - Audit Tome 10 (3): 47- 67.

Ang, James, et Pamela P. Peterson. 1984. The Leasing Puzzle. Journal of Finance 39 (4):

1055- 65. Bacqueroët, Daniel. 2010. Pas de ligne Maginot face aux IFRS. Vox-Fi - le blog de la

$D F C G$. juin

10. http://www.voxfi.fr/

Barbe, Odile. 2012. Projet de Directive comptable relatif aux états financiers : quelles conséquences pour le PCG ? Vox-Fi - le blog de la DFCG. avril 7. http://www.voxfi.fr/ 
Carmona, Salvador, et Marco Trombetta. 2008. On the global acceptance of IAS/IFRS accounting standards: The logic and implications of the principles-based system. Journal of Accounting and Public Policy, International Financial Reporting Standards, 27 (6): 455- 61.

Colasse, Bernard. 1997. The French notion of the image fidele: the power of words. European Accounting Review 6 (4): 681- 91.

Colasse, Bernard, et Peter Standish. 1998. De la réforme 1996-1998 du dispositif français de normalisation comptable. Comptabilité - Contrôle - Audit 4 (2): 5- 27.

De Bodt, Eric, Marie-Christine Filareto, et Frédéric Lobez. 2000. Décision de crédit-bail, dette bancaire et risque moral = Decision of financial leasing, banking debt and moral risk. Laboratoire de recherches économiques et sociales. Document de travail.

Delesalle, Éric. 2014. Abécédaire de vingt-six lettres comptables. Les Petites Affiches 189 (septembre): 4- 8.

Farjat, Gérard. 1986. L'importance d'une analyse substantielle en droit économique. Revue internationale de droit économique 1: 9-42.

Garnier, Pierre. 1947. Technique comptable approfondie: La Comptabilité. Algèbre du droit et méthode d'observation des sciences économiques. Paris: Dunod.

Gélard, Gilbert. 2013. La nouvelle Directive comptable européenne. DFCG News - Vox-Fi. octobre 16. http://dfcg-news.com/vox-fi/.

Hoarau, Christian. 1995. L'harmonisation comptable internationale. Vers la reconnaissance mutuelle normative ? Comptabilité - Contrôle - Audit. Tome 1 (2): 75- 88.

Hoarau, Christian. 2003. Place et rôle de la normalisation comptable en France. Revue française de gestion 29 (147): 33- 47.

Lagarrigue, J.P. 1983. Reflexions sur I'image fidèle: à propos de l'espèce Argyll. Revue Française de Comptabilité, no 134: 140.

Larroumet, Christian. 1984. Introduction à l'étude du droit privé. Vol. t.1. Droit Civil. Paris: Economica.

Lease, Ronald C., McConnell John J., et Schallheim James S.. 1990. Realized Returns and the Default and Prepayment Experience of Financial Leasing Contracts. FM: The Journal of the Financial Management Association 19 (2): 11- 20.

Lebrun, Benoît. 2009. La prédominance de la substance sur la forme dans les règles comptables françaises. Revue Française de Comptabilité, no 427.

Lobez, Frédéric, et Jean-Christophe Statnik. 2007. Une décision de crédit-bail peut aussi être un bon signal. Revue économique 58 (4): 941- 51.

Lopater, Claude. 2015. La connexion comptabilité-fiscalité, source d'insécurité fiscale! (1ère partie : les causes). Vox-Fi - le blog de la DFCG. juin 15. http://www.voxfi.fr/. 
Medus, Jean-Louis. 1999. De quelques problématiques contemporaines de droit comptable (1ère partie). Petites affiches, no 25 (février): 13.

Nobes, Christopher. 2006. The survival of international differences under IFRS: towards a research agenda. Accounting and business research 36 (3): 233-245.

Pasqualini, François. 1992. Le principe de l'image fidèle en droit comptable, 1re édition. Litec.

Raffournier, Bernard. 2007. Les oppositions françaises à l'adoption des IFRS : examen critique et tentative d'explication. Comptabilité Contrôle Audit 3 (13): 21- 41.

Raybaud-Turrillo, Brigitte. 1995. Droit comptable et droit économique : une approche renouvelée de la patrimonialité. Comptabilité - Contrôle - Audit Tome 1 (1): 25- 44.

Rossignol, Jean-Luc. 2007. Le juge de l'impôt face aux normes comptables internationales. Comptabilité - Contrôle - Audit Tome 13 (3): 113- 28. doi:10.3917/cca.133.0113.

Schallheim, James, Wells Kyle, et Whitby Ryan J.. 2013. Do leases expand debt capacity? Journal of Corporate Finance 23 (décembre): 368- 81. doi:10.1016/j.jcorpfin.2013.09.004.

Schmit, Mathias. 2004. Credit risk in the leasing industry. Journal of Banking \& Finance, Retail Credit Risk Management and Measurement, 28 (4): 811- 33.

Senicourt, Patrick. 2012. Du "bilan fiscal" au "bilan économique" : vers plus de relief dans les comptes annuels. Revue française de comptabilité, no 457 (septembre): 27- 31.

Sériaux, Alain. 1994. La notion juridique de patrimoine. Brèves notations civilistes sur le verbe avoir. Revue Trimestrielle de Droit Civil (RTD Civ) 4 (janvier): 801- 13.

Simon, François-Xavier. 2010. IFRS pour PME : un débat à ouvrir. Vox-Fi - le blog de la DFCG. mars 22. http://www.voxfi.fr/.

Tort, Eric. 2012. La complexité comptable à l'épreuve des faits. Revue Gestion 2000 : management et prospective 29 (5): 45. 


\section{Annexe 1 : exemple de traitement fiscal d'un crédit-bail du point de vue du bailleur}

Le tableau montre le calcul du loyer et du résultat fiscal du bailleur pour un matériel de transport d'une valeur en début de contrat de 100000 euros, et d'une valeur résiduelle de 10000 euros, dont on suppose qu'il s'agit de la valeur de levée d'option. Le modèle retraité envisage une activation du contrat dans les comptes sociaux et la reconnaissance en produits du seul intérêt financier. Dans les 4 modèles l'impôt payé cumulé est le même, mais le différé lié à l'amortissement du bien améliore le taux de rendement interne de l'opération. 
Valeur actuelle $100000,00 €$

Valeur résiduetle $10000,00 €$

taux

Durée bail

-

\begin{tabular}{|c|c|c|c|c|c|c|}
\hline \multirow[t]{2}{*}{ Inée } & \multicolumn{2}{|r|}{$\begin{array}{l}\text { Dette début } \\
\text { période }\end{array}$} & \multirow{2}{*}{$\begin{array}{l}\text { Loyer } \\
\begin{array}{l}9170,81 €\end{array}\end{array}$} & $\begin{array}{l}\text { Amort. } \\
\text { Fiscal }\end{array}$ & \multicolumn{2}{|c|}{$\begin{array}{l}\text { IS (-charge, } \\
\text { + produit) Flux net }\end{array}$} \\
\hline & & $100000,00 €$ & & $-4170,81 €$ & $-1666,67 €$ & $92495,86 €$ \\
\hline & 2 & $95829,19 €$ & $9170,81 €$ & $-4379,35 €$ & $-1597,15 €$ & $7573,66 €$ \\
\hline & 3 & $91449,85 €$ & $9170,81 €$ & $-4598,31 €$ & $-1524,16 €$ & $7646,65 €$ \\
\hline & & $86851,53 €$ & $9170,81 €$ & $-4828,23 €$ & $-1447,53 €$ & $7723,28 €$ \\
\hline & & $82023,31 € 9$ & $170,81 €$ & $-5069,64 €$ & $-1367,06 €$ & $7803,75 €$ \\
\hline & 6 & $76953,66 €$ & $9170,81 €$ & $-5323,12 €$ & $-1282,56 €$ & $7888,25 €$ \\
\hline & 7 & $71630,54 €$ & $9170,81 €$ & $-5589,28 €$ & - $1193,84 €$ & $7976,97 €$ \\
\hline & -8 & $66041,26 €$ & $9170,81 €$ & $-5868,74 €$ & $-1100,69 €$ & $8070,12 €$ \\
\hline & वै 9 & $60172,52 € 9$ & $170,81 €$ & $-6162,18 €$ & $-1002,88 €$ & $8167,93 €$ \\
\hline & 10 & $54010,34 €$ & $9170,81 €$ & $-6470,29 €$ & - $900,17 €$ & $8270,64 €$ \\
\hline & 11 & $47540,05 €$ & $9170,81 €$ & $-6793,80 €$ & - $792,33 €$ & $8378,48 €$ \\
\hline & 12 & $40746,25 €$ & $9170,81 €$ & $-7133,49 €$ & $679,10 €$ & $8491,71 €$ \\
\hline & 13 & $33612,76 €$ & $9170,81 €$ & $-7490,17 €$ & - $\quad 560,21 €$ & $8610,60 €$ \\
\hline & 14 & $26122,59 €$ & $9170,81 €$ & $-7864,68 €$ & - $435,38 €$ & $8735,43 €$ \\
\hline & 15 & $18257.91 €$ & $9170,81 €$ & $-8257.91 €$ & $304,30 €$ & $18866,51 €$ \\
\hline
\end{tabular}

$18257,91 € 9170,81$ $10000,00 €$

\begin{tabular}{|c|c|c|c|}
\hline \multicolumn{2}{|c|}{ Modèle fiscal 1} & \multicolumn{2}{|c|}{ Modèle fiscal 2} \\
\hline Durée & 15 & Durée & 15 \\
\hline Régime & Financier & Régime & Linéaire \\
\hline TRI & $3,82 \%$ & TRI & \\
\hline
\end{tabular}

Amort. IS (-charge,

Fiscal + produit) Flux net

$-6000,00 €-1056,94 €-91886,13$ $-6000,00 €-1056,94 € \quad 8113,87 €$ $-6000,00 €-1056,94 € \quad 8113,87 €$ $-6000,00 €-1056,94 € \quad 8113,87 €$ $-6000,00 €-1056,94 € \quad 8113,87 €$ $-6000,00 €-1056,94 € \quad 8113,87 €$ $-6000,00 €-1056,94 € \quad 8113,87 €$ $-6000,00 €-1056,94 € \quad 8113,87 €$ $-6000,00 €-1056,94 € \quad 8113,87 €$ $-6000,00 €-1056,94 € \quad 8113,87 €$ 8113,87 - $6000,00 €-1056,94 € \quad 8113,87$ $-6000,00 €-1056,94 € \quad 8113,87$ $-6000,00 €-1056,94 € \quad 8113,87 €$ $-6000,00 €-1056,94 € \quad 8113,87 €$ $-6000,00 €-1056,94 € \quad 18113,87 €$

\begin{tabular}{|l|r|}
\hline \multicolumn{2}{|c|}{ Modèle fiscal 3 } \\
\hline Durée & 8 \\
Régime & Dégressif \\
\hline TRI & $4,84 \%$ \\
\hline
\end{tabular}

IS (-charge,

Amort. Fiscal + produit) Flux net $28125,00 € \quad 6318,06 €-84511,13 €$ $-20214,84 € \quad 3681,35 € 12852,16 €$ $-14529,42 € \quad 1786,20 € \quad 10957,01 €$ $\begin{array}{lll}-10443,02 € & 424,07 € & 9594,88 €\end{array}$ $-7505,92 €-\quad 554,96 € \quad 8615,85 €$ 3060,60€-2036,74€ $7134,07 €$ \begin{tabular}{|l|l|}
\hline $3060,60 €-2036,74 €$ & $7134,07 €$ \\
\hline
\end{tabular} - $3060,60 €-2036,74 € \quad 7134,07 €$ \begin{tabular}{l|l}
$€-3056,94 €$ & $6113,87 €$
\end{tabular} € $-3056,94 € \quad 6113,87 €$ €-3056,94€ $6113,87 €$ \begin{tabular}{ll|l}
$€-3056,94 €$ & $6113,87 €$
\end{tabular} €-3056,94€ $6113,87 €$ €-3 056,94€ 6113,87 €- $-3056,94 € \quad 16113,87 €$

\begin{tabular}{|l|r|}
\hline \multicolumn{2}{|c|}{ Modèle retraité } \\
\hline Durée & 15 \\
\hline Régime & Activation \\
\hline TRI & $3,82 \%$ \\
\hline
\end{tabular}
$3,82 \%$

$105000,00 €-100000,00 €-1666,67 €-92495,86 €$ \begin{tabular}{|l|l|l|}
\hline $4791,46 €$ & $-1597,15 €$ & $7573,66 €$ \\
\hline
\end{tabular} \begin{tabular}{lll|l}
$4572,49 €$ & $-1524,16 €$ & $7646,65 €$ \\
\hline
\end{tabular} \begin{tabular}{lll|l}
$4342,58 €$ & $-1447,53 €$ & $7723,28 €$
\end{tabular} \begin{tabular}{|l|l|l|}
\hline $4101,17 €$ & $-1367,06 €$ & $7803,75 €$ \\
\hline
\end{tabular} \begin{tabular}{|l|l|l|}
\hline $3847,68 €$ & $-1282,56 €$ & $7888,25 €$ \\
\hline
\end{tabular} \begin{tabular}{|l|l|l|}
\hline $3581,53 €$ & $-1193,84 €$ & $7976,97 €$ \\
\hline
\end{tabular} \begin{tabular}{l|l|l}
$3302,06 €$ & $-1100,69 €$ & $8070,12 €$
\end{tabular} $3008,63 € \quad-1002,88 € \quad 8167,93 €$ $2700,52 €-900,17 € \quad 8270,64 €$ \begin{tabular}{|l|l|l|}
$23377,00 €$ & $-792,33 €$ & $8378,48 €$ \\
\hline
\end{tabular} \begin{tabular}{|l|l|l|}
$2037,31 €$ & $-679,10 €$ & $8491,71 €$
\end{tabular} \begin{tabular}{|l|l|l|}
\hline $1680,64 €$ & $-560,21 €$ & $8610,60 €$ \\
\hline
\end{tabular} \begin{tabular}{|l|l|l}
$1306,13 €$ & $-435,38 €$ & $8735,43 €$
\end{tabular} $912,90 €-304,30 € \quad 18866,51 €$ 